\title{
Intervenciones coronarias percutáneas guiadas con medición de flujo de reserva coronario.
}

\author{
Mauricio Aninat, Humberto Torres, Luis Becerra, Felipe Aninat, Patricia Valenzuela, \\ Sergio Ramos, Alex Mora
}

\author{
Laboratorio de Hemodinamia y Angiografía. \\ Hospital Dr. Gustavo Fricke. Viña del Mar. \\ Universidad de Valparaiso, Facultad de Medicina, \\ Escuela de Medicina, Valparaíso, Chile.
}

\section{Resumen:}

Introducción: La revascularización de una estenosis coronaria que induce isquemia mejora el estatus funcional del paciente y su pronóstico. Por el contrario, la angioplastía de una lesión que no provoca isquemia puede implicar complicaciones sin beneficios. La medición de flujo de reserva coronario (FFR) es un índice del significado fisiopatológico de las estenosis coronarias.

Objetivos: Comparar los eventos cardiovasculares adversos mayores (MACE) en pacientes en quienes la FFR permitió excluir una o más lesiones de angioplastia (Grupo FFR >0,75) con aquellos en que ésta obligó a la revascularización (grupo FFR $<0,75$ ).

Métodos: Estudio observacional en 74 pacientes derivados para angioplastía entre agosto de 2006 y julio de 2010. Se realizó medición de FFR con adenosina a lesiones de severidad intermedia (60-70\%). Una FFR translesional $<0,75$ se consideró significativa para isquemia y subsidiaria de angioplastía.

Se hizo un seguimiento de los MACE definidos como: muerte, infarto, necesidad de revascularización $\mathrm{y} / \mathrm{o}$ presencia de angor.
Resultados: 35 pacientes $(47,3 \%)$ constituyeron el Grupo FFR >0,75, y en ellos disminuyó el porcentaje de lesiones múltiples angiográficamente significativas (dos o más vasos), desde un 51,4\% (previo a la realización de FFR), a un $25,7 \%$. En este grupo se utilizó un promedio de 0.7 stents por paciente, mientras que en el grupo FFR $<0,75$ (39 pacientes), este fue de 1.5 stents. Se efectuó un seguimiento promedio de 21,5 meses. Diecinueve pacientes $(25,6 \%)$ desarrollaron MACE (28 eventos). No hubo fallecidos ni diferencias significativas en el análisis multifactorial en cuanto a infartos o angor, pero sí en la necesidad de nueva revascularización, siendo ésta significativamente mayor en el grupo FFR $<0,75$ (12,8\% versus $2,9 \%, \mathrm{p}=0,047)$.

Conclusiones: La medición del FFR permite, en caso de descartar isquemia, disminuir la necesidad de angioplastías, sin aumento de MACE en el seguimiento a mediano plazo. En el grupo de pacientes con FFR $<0,75$ se requieren nuevos procedimientos de revascularización, lo que es concordante con el mayor número de lesiones tratadas.

\section{Correspondencia:}

Dr. Mauricio Aninat Harnecker,

maraninat@gmail.com 
Background: The treatment of coronary stenosis causing myocardial ischemia improves functional capacity and prognosis. Treatment of non-ischemia inducing coronary stenosis may lead to complications with no benefit to the patient. Measurement of coronary flow reserve (CFR) may be used to assess the significance of coronary artery stenosis.

Aim: To compare major adverse cardiovascular events (MACE) in patients with significant stenosis (CFR < 0.75 ), with those in which one or more stenosis was not significant (CFR $>0.75$ ) and thus were not subjected to PTCA in the corresponding artery.

Methods: 74 patients were included from August 2006 to July 2010. CFR was measured in lesions exhibiting 60$70 \%$ stenosis, using adenosine. A value $<0.75$ was considered significant and led to PTCA. Patients were followed for death, myocardial infarction, revascularization and/or angina.

Results: 35 patients (47\%) constituted the $>0.75$ CFR group. After evaluation of CFR the number of significant coronary stenosis decreased from $51.4 \%$ to $25.7 \%$. They received a mean of 0.7 stents per patient. On the other hand, in the group with CFR $<0.75$ ( 39 patients) the mean number of stents was 1.5. Patients were followed for a mean of 21.5 months. 28 MACE events were observed in 19 patients $(25.6 \%)$. No deaths were observed. Multivariable analysis revealed no significant difference between groups regarding myocardial infarction or angina. The need for myocardial revascularization was greater in the CFR $<0.75$ group compared to the CFR $>0.75$ group ( $12.8 \%$ vs $2.9 \%$, respectively, $\mathrm{p}=0.047$ ).

Conclusion: The exclusion of myocardial ischemia as inferred by a CFR $>0.75$ allowed a significantly lower number of PTCAs without increase in MACE at a medium term follow up. A greater number of revascularization procedures was required in patients with CFR $<0.75$, which is expected from the greater number of lesions subjected to PTCA.

Key words: PTCA, coronary stenosis, coronary flow reserve

\section{Introducción}


medición del FFR revela que no produce isquemia, ésta no debe ser sometida a angioplastía.

\section{Material y Métodos}

Entre Agosto del año 2006 y Julio del 2010, se realizó evaluación de la importancia fisiológica de las lesiones coronarias de severidad intermedia (apreciación visual de la estenosis entre 50 y $70 \%$ ) empleando la medición del FFR, a 74 pacientes enviados para angioplastía coronaria en base a los hallazgos angiográficos. Para ello se utilizó una guía de presión (Sistemas Médicos RADI).

Este alambre-guía coronario de 0,014 pulgadas de diámetro contiene en su parte distal un transductor electrónico de presión, y es introducido a través del catéter guía dentro de la coronaria, negociando el paso a través de la estenosis y ubicando la zona del transductor distal a ella. A continuación, se inyecta adenosina intracoronaria por el catéter guía en dosis variables entre 30 y 60 microgramos; ello produce una vasodilatación máxima de la microcirculación coronaria; condición indispensable para la medición del gradiente de presiones translesionales. Se miden en forma simultánea las presiones obtenidas en el extremo del catéter guía y en el transductor de la guía. El FFR es calculado con la presión media distal a la estenosis, dividida por la presión media aórtica obtenida en forma simultánea durante la hiperemia máxima. Se considera que una isquemia es significativa cuando el FFR es $\leq$ a 0,75, lo que significa que la estenosis impide, en vasodilatación máxima, un $25 \%$ o más del flujo miocárdico máximo que le correspondería en caso de no existir dicha estenosis. Un FFR sobre 0,75 supone ausencia de isquemia.

La medición del FFR se efectuó sólo en las lesiones angiográficas de severidad intermedia, en el rango de $50 \%$ a $70 \%$ por estimación visual de 2 operadores. Si el FFR resultaba igual o menor de 0,75 , se procedía a la angioplastía de la lesión. Si, por el contrario, el FFR era mayor de 0,75 no se efectuaba intervención en esa lesión. Los pacientes incluidos en este estudio presentaron lesiones coronarias de un vaso o de múltiples vasos y fueron catalogados clínicamente como anginosos estables. Las angioplastías se efectuaron con las técnicas habituales utilizando la guía de presión para la introducción de balones y stents. En la gran mayoría se utilizó stents sin drogas.

Identificados los pacientes, se procedió a la recolección de datos mediante entrevista telefónica y revisión de fichas clínicas. Los puntos finales que se registraron fueron: muerte, infarto, revascularización y presencia de angina

Para efecto de análisis estadístico se utilizó el programa SPSS.

\section{Resultados}

Desde Agosto del año 2006 hasta Julio del año 2010, se revisaron en forma consecutiva los procedimientos efectuados en nuestro Laboratorio en los cuales se utilizó la medición del FFR en lesiones angiográficas intermedias para dilucidar si producían o no isquemia del área de miocardio irrigado. Ello permitió reunir a 74 pacientes, 54 hombres y 20 mujeres, con edades promedio de $64,5 \pm 9,3$ años. 76 estenosis coronarias fueron evaluadas con la guía de presión. Como resultado de la medición del FFR se observó una reducción importante del grado isquémico lesional coronario del conjunto de los pacientes. La enfermedad multivaso disminuyó desde un $51,4 \%$ a un $25,7 \%$, y un $19 \%$ de los pacientes resultó sin lesiones significativas (Gráficos 1 y 2).
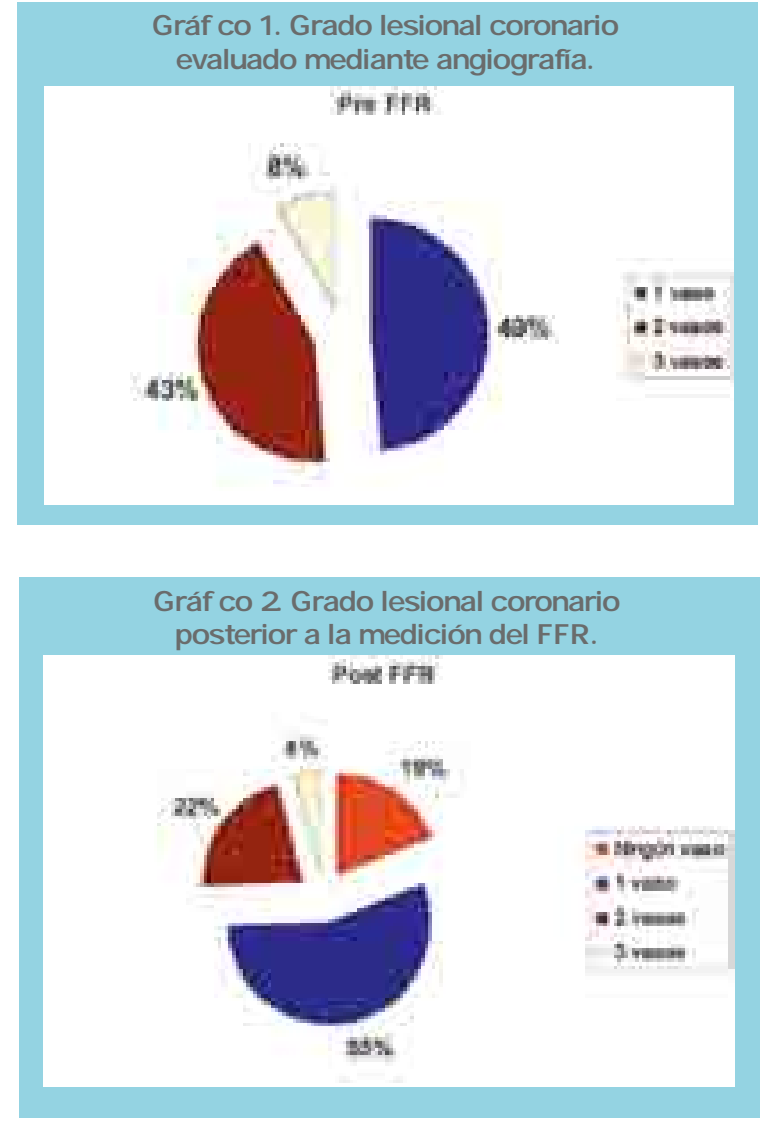

De acuerdo al resultado de la medición del FFR los pacientes se separaron en 2 Grupos. El Grupo 1 lo constituyeron 35 pacientes (47.3\% del total) cuya medición 


\section{Figura 1. Distribución de los pacientes según resultados de la medición del FFR.}
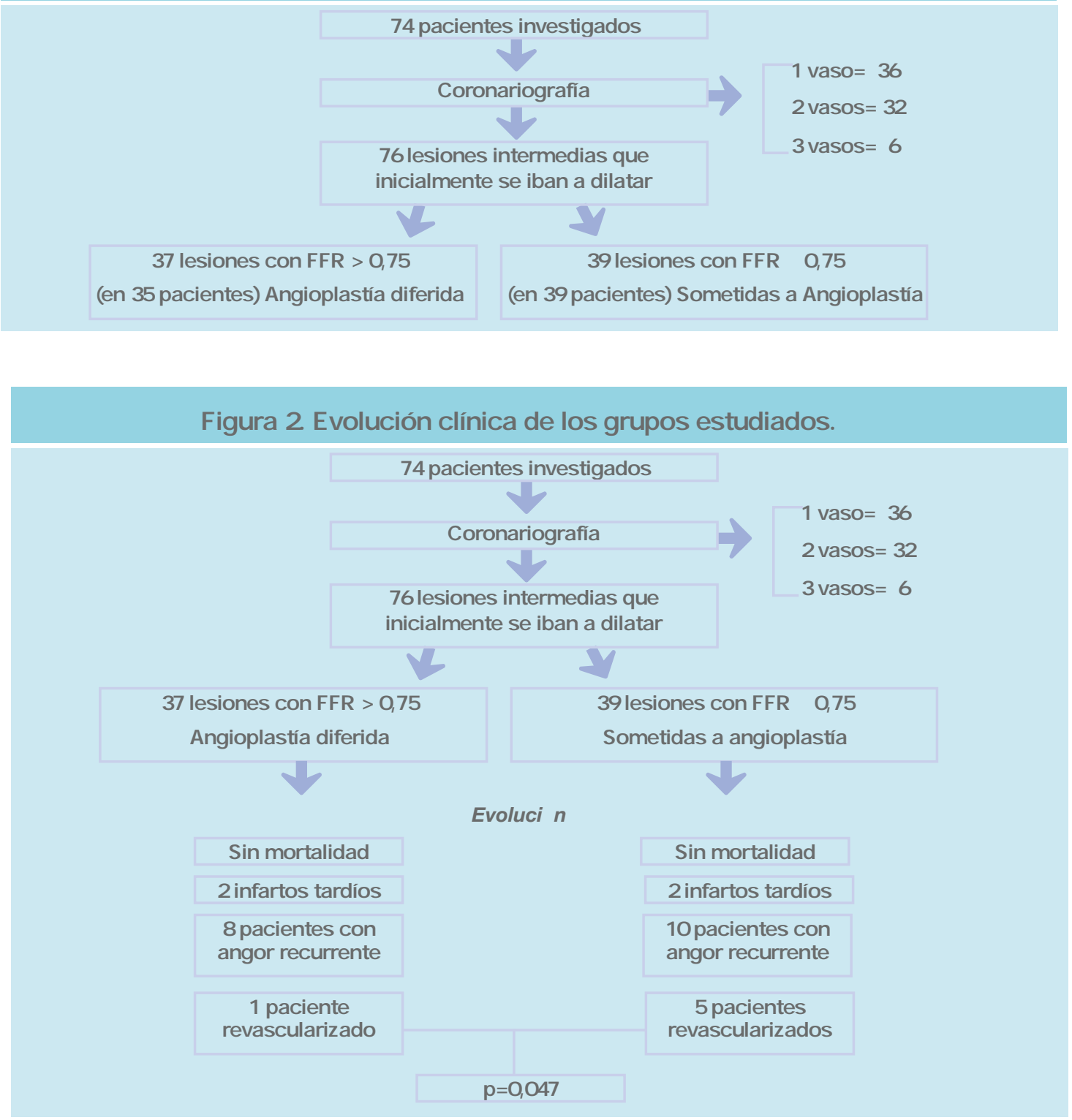

del FFR fue mayor de 0,75 y por lo tanto no requirieron intervención sobre las lesiones coronarias evaluadas. El Grupo 2 lo conformaron 39 pacientes (52.7\% del total) con FFR $\leq$ a 0,75 a los cuales se les efectuó angioplastía con implante de stent en la lesión evaluada (Figura1); sólo 6 de estos pacientes recibieron stent con drogas $(15,3 \%)$. Todos los procedimientos fueron exitosos.

Los aspectos demográficos, factores de riesgo coronarios y grado lesional coronario fueron similares para ambos grupos (Tabla 1).

La reducción de las lesiones significativas como resultado de la medición del FFR repercutió en una disminución importante de las angioplastías en el Grupo 1. En este grupo se utilizó 0.66 stents por paciente y en el Grupo 2 se emplearon 1.46 stents por paciente. El tiempo de seguimiento clínico promedio fue de 21,5 \pm 15,1 meses. En cuanto a los puntos finales del estudio, se observó que no hubo diferencias en los grupos en relación a mortalidad, infartos e isquemia residual. Sin embargo, se apreció una diferencia significativa en la necesidad de una nueva revascularización en favor del grupo 1 (Figura 2).

\section{Discusión}

El presente estudio muestra que la medición objetiva 


\begin{tabular}{|c|c|c|c|}
\hline & Grupo 1 & Grupo 2 & $\mathbf{p}$ \\
\hline Edad (años +/- DE) & $66,6+/-9,3$ & $62,7+/-8,9$ & ns \\
\hline Hombres (n) & $24(69 \%)$ & $30(77 \%)$ & ns \\
\hline \multicolumn{4}{|c|}{ Factores de riesgo (n) } \\
\hline DM & $14(40 \%)$ & $16(41 \%)$ & ns \\
\hline HTA & $31(89 \%)$ & 35 (90\%) & ns \\
\hline Tabaquismo & $11(31 \%)$ & $12(31 \%)$ & ns \\
\hline Dislipidemia & $12(34 \%)$ & $9(23 \%)$ & ns \\
\hline \multicolumn{4}{|l|}{ Vasos enfermos (n) } \\
\hline Un vaso 1 & $3(37 \%)$ & $23(59 \%)$ & ns \\
\hline Multivaso & $22(63 \%)$ & $16(41 \%)$ & ns \\
\hline
\end{tabular}

del carácter isquémico de las lesiones coronarias angiográficas intermedias, permite reducir el número de angioplastías que se programan guiadas por angiografía. Esto se logra en el Laboratorio de Hemodinamia, en forma expedita, empleando la guía de presión sin demorar el procedimiento ya que se emplea la misma guía en caso de requerirse la angioplastía. En esta serie no se apreciaron diferencias en mortalidad, infartos o recurrencia de angina, pero se observaron menos reintervenciones en el grupo en que las lesiones no se intervinieron. Estimamos que el número reducido de pacientes, el escaso grado de compromiso coronario (la mayoría con lesión de 1 vaso) y quizás el poco tiempo de observación, no permitió observar otras diferencias, como está consignado en la literatura ${ }^{7}$. El número de stents ocupados se redujo en forma importante, con la consiguiente

\section{Referencias:}

1. ISKANDER SH, ISKANDRIAN AE. Risk assesment using single-photon emission computed tomographic technetium- $99 \mathrm{~m}$ setamibi imaging. J Am Coll Cardiol 1998; 32: 57-62.

2. HACHAMOVITCH R, HAYES SW, FRIEDMAN JD, COHEN I, BERMAN DS. Comparison of the Short-term survival benefit associated with revascularization compared with medical therapy in patients with no prior coronary artery disease undergoing sterss myocardial perfusion Circulation 2003;107:29002907.

3. PIJLS NH, VAN SCHAARDENBURGH P, MANOHARAN P, BOERSMAE, BECH JW, VANT VEER M, et al. Percutaneous coronary intervention of functionally nonsignificant stenosis: 5-year follow-up of the DEFER study. J Am Coll Cardiol. 2007; 49: $2105-11$ disminución de costos.

En nuestro estudio solamente fueron evaluadas con FFR las lesiones angiográficamente intermedias, destacando, en relación a otros estudios ${ }^{3}$, la alta proporción de lesiones isquémicas detectadas con la guía de presión (57\%) lo que revela una buena aproximación visual angiográfica de la severidad de las lesiones.

En conclusión, la angioplastía coronaria de lesiones angiográficas intermedias guiada con medición del flujo fraccional de reserva coronario, permite reconocer a aquellas que no producen isquemia y que, por lo tanto, no deben ser revascularizadas. La evolución a mediano plazo de estos pacientes es similar a la de aquellos sometidos a angioplastía en cuanto a mortalidad, infartos y angina recurrente, pero es superior en relación a la menor necesidad de nuevas revascularizaciones.
4. FISCHER JJ, SAMADY H, MCPHERSON JA, SAREMBOCK IJ, POWERS ER, GIMPLE LW, Et al. Comparison between visual assesment and quantitative angiography versus fractional flow reserve for native coronary narrowings of moderate severity. Am J Cardiol 2002; 90: 210-5.

5. LIMA RS, WATSON DD, GOODE AR, SIADATY MS, RAGOSTA M, BELLER GA, et al. Incremental value of combined perfusion and function over perfusion alone by gated SPECT myocardial perfusion imaging for detection of severe three-vessel coronary artery disease. J Am Coll Cardiol. 2003;42:64-70

6. PIJLS NH, VAN GELDER B, VAN DER VOORT P, PEELS K, BRACKE FA, BONNIER HJ, et al. Fractional flow reserve. A useful index to evaluate the influence of an epicardial coronary stenosis on myocardial blood flow. Circulation. 1995;92:3183-93 et al. Fractional Flow Reserve versus Angiography for Guiding Percutaneous Coronary Intervention. N Engl J Med 2009; 360:213-24. 